Commun. Fac. Sci. Univ. Ank. Ser. A1 Math. Stat.

Volume 68, Number 2, Pages 2313-2323(2019)

DOI: $10.31801 /$ cfsuasmas. 558169

ISSN 1303-5991 E-ISSN 2618-6470

http://communications.science.ankara.edu.tr/index.php?series=A1

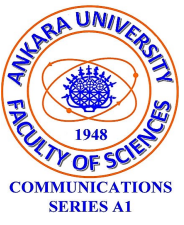

\title{
APPROXIMATION PROPERTIES OF BERNSTEIN-KANTOROVICH TYPE OPERATORS OF TWO VARIABLES
}

\author{
DÖNE KARAHAN AND AYDIN IZGI
}

\begin{abstract}
In this study, the generalized Bernstein-Kantorovich type operators are introduced and some approximation properties of these operators are studied in the space of continuous functions of two variables on a compact set. The rate of convergence of these operators are obtained by means of the modulus of continuity. The Voronovskaya type theorem is given and some differential properties of these operators are proved.
\end{abstract}

\section{INTRODUCTION}

In 1912 , for a function $f$ defined on the closed interval $[0,1]$, the expression

$$
B_{n}(f ; x)=\sum_{k=0}^{n} f\left(\frac{k}{n}\right)\left(\begin{array}{l}
n \\
k
\end{array}\right) x^{k}(1-x)^{n-k}
$$

was called the Bernstein polynomial of order $n$ of the function $f$ in [1]. In [2], Korovkin's theorem sometimes also called Bohman-Korovkin theorem, arose from the study of the role of Bernstein polynomials in the proof of the Weierstrass approximation theorem. Later, the various generalizations of Bernstein polynomials (1.1) were investigated in [3]-9]. In 1930, L.V. Kantorovich constructed and studied the linear positive operators $K_{m}: L_{1}([0,1]) \rightarrow C([0,1])$ defined by

$$
\left(K_{m} f\right)(x)=(m+1) \sum_{k=0}^{m} p_{m, k}(x) \int_{\frac{k}{m+1}}^{\frac{k+1}{m+1}} f(s) d s .
$$

The operators 1.2 are known as the Kantorovich operators. These operators are obtained from the classical Bernstein operators (1.1). In [9], Kahvecibasi studied approximation properties of generalized Bernstein-Kantorovich operators on closed interval $[-1,1]$.

Received by the editors: April 26, 2019; Accepted: July 09, 2019.

2010 Mathematics Subject Classification. 41A25, 41A36.

Key words and phrases. Bernstein-Kantorovich operators, modulus of continuity, Voronovskaya type theorem.

(C)2020 Ankara University Communications Faculty of Sciences University of Ankara-Series A1 Mathematics and Statistics 
There are many investigations devoted to the problem of approximating continuous functions by classical Bernstein polynomials, as well as by two dimensional Bernstein polynomials and their generalizations. We refer to papers [1]-[14].

In this note, inspired by the operators 1.1 and 1.2 , we consider generalized Bernstein-Kantorovich operators for functions of two variables. To this end, let $f \in C(\mathbb{A})$, where $\mathbb{A}=[-1,1] \times[-1,1]$ and define the linear positive operators $D_{n, m}(f ; x, y), n, m \in \mathbb{N}$ in the following way:

$$
D_{n, m}(f ; x, y)=\frac{n+1}{2} \frac{m+1}{2} \sum_{k=0}^{n} \sum_{j=0}^{m} \phi_{n, m}^{k, j}(x, y) \int_{2 \frac{k}{n+1}-1}^{2 \frac{k+1}{n+1}-1} \int_{2 \frac{j}{m+1}-1}^{2 \frac{j+1}{m+1}-1} f(t, u) d t d u
$$

with

$$
\phi_{n, m}^{k, j}(x, y)=\varphi_{n, k}(x) \varphi_{m, j}(y)
$$

and

$$
\varphi_{n, k}(x)=\frac{1}{2^{n}}\left(\begin{array}{l}
n \\
k
\end{array}\right)(1+x)^{k}(1-x)^{n-k} .
$$

As usual, let $C(\mathbb{A})$ be the the space of all real valued continuous functions on $\mathbb{A}$ endowed with the norm

$$
\|f\|_{(\mathbb{A})}=\max _{(x, y) \in \mathbb{A}}|f(x, y)|
$$

Let $f \in C(\mathbb{A})$.The full modulus of continuity of $f$ is defined as follows:

$$
\omega(f, \delta)=\underset{\frac{\max }{\sqrt{\left(x_{1}-x_{2}\right)^{2}+\left(y_{1}-y_{2}\right)^{2}}} \leq \delta}{(x, y) \in \mathbb{A}}
$$

Partial modulus of continuity with respect to $x$ and $y$ is defined by

$$
\begin{array}{ccc}
\omega^{(1)}(f, \delta)= & \max _{\left(x_{1}, y\right),\left(x_{2}, y\right) \in \mathbb{A}}\left|f\left(x_{1}, y\right)-f\left(x_{2}, y\right)\right|, \\
\left|x_{1}-x_{2}\right| \leq \delta & \\
\omega^{(2)}(f, \delta)= & \max _{\substack{\left(x, y_{1}\right),\left(x, y_{2}\right) \in \mathbb{A} \\
\left|y_{1}-y_{2}\right| \leq \delta}}\left|f\left(x, y_{1}\right)-f\left(x, y_{2}\right)\right|,
\end{array}
$$

respectively. It is known that the full and partial modulus of continuity satisfy the following properties:

$$
\omega(f, \delta) \leq(1+\lambda) \omega(f, \delta), \quad \lim _{\delta \rightarrow 0} \omega(f, \delta)=0 .
$$




\section{MAIN RESULTS}

In this section we give some classical approximation properties of the operators $D_{n, m}$ on the set $\mathbb{A}$.

Then by simple calculations, one can obtain the following lemmas.

Lemma 2.1. For $\forall(x, y) \in \mathbb{A}$ and $\forall n, m \in \mathbb{N}$, Bernstein-Kantorovich operators 1.3) satisfy the following equalities:

$$
\begin{gathered}
D_{n, m}(1 ; x, y)=1 \\
D_{n, m}(t ; x, y)=x-\frac{x}{n+1}, \\
D_{n, m}(u ; x, y)=y-\frac{y}{m+1}, \\
D_{n, m}\left(t^{2}+u^{2} ; x, y\right)=x^{2}-\frac{3 n x^{2}+x^{2}-n-\frac{1}{3}}{(n+1)^{2}}+y^{2}-\frac{3 m y^{2}+y^{2}-m-\frac{1}{3}}{(m+1)^{2}}, \\
D_{n, m}\left(t^{3}+u^{3} ; x, y\right)=x^{3}-\frac{x^{3}+6 n^{2} x^{3}+3 n x^{3}-3 n^{2} x+6 n+7 n x+6 n x^{2}}{(n+1)^{3}} \\
+y^{3}-\frac{y^{3}+6 m^{2} y^{3}+3 m y^{3}-3 m^{2} y+6 m+7 m y+6 m y^{2}}{(m+1)^{3}}, \\
D_{n, m}\left(t^{4}+u^{4} ; x, y\right)=x^{4}-\frac{10 n^{3} x^{4}-5 n^{2} x^{4}+10 n x^{4}-x^{4}+6 n^{3} x^{2}+6 n x^{2}}{(n+1)^{4}} \\
+\frac{10 n^{2} x^{2}+4 n x-3 n^{2}-4 n-\frac{1}{5}}{(n+1)^{4}} \\
+y^{4}-\frac{10 m^{3} y^{4}-5 m^{2} y^{4}+10 m y^{4}-y^{4}+6 m^{3} y^{2}+6 m y^{2}}{(m+1)^{4}} \\
+\frac{10 m^{2} y^{2}+4 m y-3 m^{2}-4 m-\frac{1}{5}}{(m+1)^{4}}
\end{gathered}
$$

From Lemma 2.1, we obtained following lemmas.

Lemma 2.2. For the operators $D_{n, m}$ in (1.3), we have

$$
\begin{gathered}
D_{n, m}\left((t-x)^{2} ; x, y\right)=\frac{3 x^{2}-3 n x^{2}+3 n+1}{3(n+1)^{2}} \\
D_{n, m}\left((u-y)^{2} ; x, y\right)=\frac{3 y^{2}-3 m y^{2}+3 m+1}{3(m+1)^{2}} \\
D_{n, m}\left((t-x)^{4} ; x, y\right)=\frac{n^{2} x^{4}+8 n x^{4}+x^{4}+44 n x^{2}+20 n^{2} x^{2}+24 n^{2} x+24 n^{2} x^{3}}{(n+1)^{4}} \\
+\frac{24 n x^{3}+20 n x+2 x^{2}+3 n^{2}+4 n+\frac{1}{5}}{(n+1)^{4}}
\end{gathered}
$$




$$
\begin{aligned}
D_{n, m}\left((u-y)^{4} ; x, y\right) & =\frac{m^{2} y^{4}+8 m y^{4}+y^{4}+44 m y^{2}+20 m^{2} y^{2}+24 m^{2} y+24 m^{2} y^{3}}{(m+1)^{4}} \\
& +\frac{24 m y^{3}+20 m y+2 y^{2}+3 m^{2}+4 m+\frac{1}{5}}{(m+1)^{4}}
\end{aligned}
$$

Lemma 2.3. For every fixed $\left(x_{0}, y\right) \in \mathbb{A}$ there exists a positive constant $M_{1}\left(x_{0}\right)$ such that for $n \in \mathbb{N}, D_{n, n}\left(\left(t-x_{0}\right)^{4} ; x_{0}, y\right) \leq M_{1}\left(x_{0}\right) n^{-2}$.

Theorem 2.1. (See [10] If $\left(T_{n, m}\right)$ is a sequence of linear positive operators satisfying the conditions

$$
\begin{array}{r}
\lim _{n, m \rightarrow \infty}\left\|T_{n, m}(1 ; x, y)-1\right\|_{C(\mathbb{X})}=0 \\
\lim _{n, m \rightarrow \infty}\left\|T_{n, m}(t ; x, y)-x\right\|_{C(\mathbb{X})}=0 \\
\lim _{n, m \rightarrow \infty}\left\|T_{n, m}(u ; x, y)-y\right\|_{C(\mathbb{X})}=0 \\
\lim _{n, m \rightarrow \infty} \| T_{n, m}\left(\left(t^{2}+u^{2} ; x, y\right)-\left(x^{2}+y^{2}\right) \|_{C(\mathbb{X})}=0\right.
\end{array}
$$

then for any function $f \in C(\mathbb{X})$, which is bounded in $\mathbb{R}^{2}$,

$$
\lim _{n, m \rightarrow \infty}\left\|T_{n, m}(f ; x, y)-f(x, y)\right\|_{C(\mathbb{X})}=0,
$$

where $\mathbb{X}$ is a compact set.

In the following theorem we show that the linear positive operator $D_{n, m}$ in 1.3 converges to $f$ uniformly with the help of Theorem 2.1 .

Theorem 2.2. For every $f \in C(\mathbb{A})$, the the operators $D_{n, m}$ defined by (1.3) converge uniformly to $f$ on the set $\mathbb{A}$ as $n, m \rightarrow \infty$.

Proof. From 2.1)-2.4, we have

$$
\begin{array}{r}
\lim _{n, m \rightarrow \infty}\left\|D_{n, m}(1 ; x, y)-1\right\|_{C(\mathbb{A})}=0 \\
\lim _{n, m \rightarrow \infty}\left\|D_{n, m}(t ; x, y)-x\right\|_{C(\mathbb{A})}=0 \\
\lim _{n, m \rightarrow \infty}\left\|D_{n, m}(u ; x, y)-y\right\|_{C(\mathbb{A})}=0 \\
\lim _{n, m \rightarrow \infty}\left\|D_{n, m}\left(t^{2}+u^{2} ; x, y\right)-\left(x^{2}+y^{2}\right)\right\|_{C(\mathbb{A})}=0 .
\end{array}
$$

Applying Theorem 2.1, we obtain the desired result.

Theorem 2.3. For $f \in C(\mathbb{A})$, the the following inequalities hold:

$$
\left\|D_{n, m}(f ; x, y)-f\right\|_{C(\mathbb{A})} \leq 2\left(\omega^{(1)}\left(f ; \frac{1}{\sqrt{n}}\right)+\omega^{(2)}\left(f ; \frac{1}{\sqrt{m}}\right)\right)
$$




$$
\left\|D_{n, m}(f ; x, y)-f\right\|_{C(\mathbb{A})} \leq 2 \omega\left(f ; \sqrt{\frac{1}{n}+\frac{1}{m}}\right)
$$

where $\omega, \omega^{(1)}$ and $\omega^{(2)}$ are given by (1.6), 1.7) and (1.8), respectively.

Proof. From 1.3 and 2.1, we can write

$$
\begin{aligned}
& \left|D_{n, m}(f ; x, y)-f(x, y)\right| \\
& \leq D_{n, m}(|f(t, y)-f(x, y)| ; x, y)+D_{n, m}(|f(t, u)-f(t, y)| ; x, y) \\
& \leq \omega^{(1)}\left(f ; \delta_{n}\right)\left[1+\frac{1}{\delta_{n}} \frac{n+1}{2} \sum_{k=0}^{n} \varphi_{n, k}(x) \int_{2 \frac{k}{n+1}-1}^{2 \frac{k+1}{n+1}-1}|t-x| d t\right] \\
& +\omega^{(2)}\left(f ; \delta_{m}\right)\left[1+\frac{1}{\delta_{m}} \frac{m+1}{2} \sum_{j=0}^{m} \varphi_{m, j}(y) \int_{2 \frac{j}{m+1}-1}^{2 \frac{j+1}{m+1}-1}|u-y| d u\right] .
\end{aligned}
$$

Applying the Cauchy-Schwartz inequality once more, we get

$$
\begin{aligned}
& \left|D_{n, m}(f ; x, y)-f(x, y)\right| \\
& \leq \omega^{(1)}\left(f ; \delta_{n}\right)\left[1+\frac{1}{\delta_{n}} \frac{n+1}{2} \sum_{k=0}^{n} \varphi_{n, k}(x)\left(\int_{2 \frac{k}{n+1}-1}^{2 \frac{k+1}{n+1}-1}|t-x|^{2} d t\right)^{\frac{1}{2}}\left(\int_{2 \frac{k}{n+1}-1}^{2 \frac{k+1}{n+1}-1} d t\right)^{\frac{1}{2}}\right] \\
& +\omega^{(2)}\left(f ; \delta_{m}\right)\left[1+\frac{1}{\delta_{m}} \frac{m+1}{2} \sum_{j=0}^{m} \varphi_{m, j}(y)\left(\int_{2 \frac{j}{m+1}-1}^{2 \frac{j+1}{m+1}-1}|u-y|^{2} d u\right)^{\frac{1}{2}}\left(\int_{2 \frac{j}{m+1}-1}^{2 \frac{j+1}{m+1}-1} d u\right)^{\frac{1}{2}}\right] .
\end{aligned}
$$

Applying the Cauchy-Schwartz inequality once more, we get

$$
\begin{aligned}
& \left|D_{n, m}(f ; x, y)-f(x, y)\right| \\
& \leq \omega^{(1)}\left(f ; \delta_{n}\right)\left[1+\frac{1}{\delta_{n}}\left(\frac{n+1}{2}\right)^{\frac{1}{2}}\left(\sum_{k=0}^{n} \varphi_{n, k}(x)\right)^{\frac{1}{2}}\left(\sum_{k=0}^{n} \varphi_{n, k}(x) \int_{2 \frac{k}{n+1}-1}^{2 \frac{k+1}{n+1}-1}|t-x|^{2} d t\right)^{\frac{1}{2}}\right] \\
& +\omega^{(2)}\left(f ; \delta_{m}\right)\left[1+\frac{1}{\delta_{m}}\left(\frac{m+1}{2}\right)^{\frac{1}{2}}\left(\sum_{j=0}^{m} \varphi_{m, j}(y)\right)^{\frac{1}{2}}\left(\sum_{j=0}^{m} \varphi_{m, j}(y) \int_{2 \frac{j}{m+1}-1}^{2 \frac{j+1}{m+1}-1}|u-y|^{2} d u\right)^{\frac{1}{2}}\right] \\
& =\omega^{(1)}\left(f ; \delta_{n}\right)\left[1+\frac{1}{\delta_{n}}\left(D_{n, m}\left((t-x)^{2} ; x, y\right)\right)^{\frac{1}{2}}\right] \\
& +\omega^{(2)}\left(f ; \delta_{m}\right)\left[1+\frac{1}{\delta_{m}}\left(D_{n, m}\left((u-y)^{2} ; x, y\right)\right)^{\frac{1}{2}}\right] .
\end{aligned}
$$


From (2.7) and 2.8, we obtain the (2.14) inequality. Now we prove the 2.15 inequality:

$$
\begin{aligned}
\left|D_{n, m}(f ; x, y)-f(x, y)\right| & \leq D_{n, m}(|f(t, u)-f(x, y)| ; x, y) \\
& \leq \omega\left(f ; \delta_{n, m}\right)\left[1+\frac{1}{\delta_{n, m}} \frac{n+1}{2} \frac{m+1}{2} \sum_{k=0}^{n} \sum_{j=0}^{m} \phi_{n, m}^{k, j}(x, y)\right. \\
& \left.\times \int_{2 \frac{k}{n+1}-1}^{2 \frac{k+1}{n+1}-1} \int_{2 \frac{j}{m+1}-1}^{\frac{j+1}{m+1}-1} \sqrt{(t-x)^{2}+(u-y)^{2}} d u d t\right] .
\end{aligned}
$$

Applying the Cauchy-Schwartz inequality once more, we get

$$
\begin{aligned}
& \left|D_{n, m}(f ; x, y)-f(x, y)\right| \\
& \leq \omega\left(f ; \delta_{n, m}\right)\left[1+\frac{1}{\delta_{n, m}}\left(\frac{n+1}{2} \frac{m+1}{2} \sum_{k=0}^{n} \sum_{j=0}^{m} \phi_{n, m}^{k, j}(x, y)\right.\right. \\
& \left.\left.\times \int_{2 \frac{k}{n+1}-1}^{2 \frac{k+1}{n+1}-1} \int_{2 \frac{j}{m+1}-1}^{2 \frac{j+1}{m+1}-1}(t-x)^{2}+(u-y)^{2} d u d t\right)^{\frac{1}{2}}\right] \\
& =\omega\left(f ; \delta_{n, m}\right)\left[1+\frac{1}{\delta_{n, m}}\left(D_{n, m}\left((t-x)^{2}+(u-y)^{2} ; x, y\right)\right)^{\frac{1}{2}}\right] .
\end{aligned}
$$

From (2.7) and 2.8), we obtain inequality 2.15).

Now, we obtain a Voronovskaya-type theorem and some differential properties for the positive linear operators $D_{n, m}$ in 1.3 for $n=m$.

Theorem 2.4. For every $f \in C^{2}(\mathbb{A})$, we have

$$
\begin{gathered}
\lim _{n \rightarrow \infty} n\left\{D_{n, n}(f ; x, y)-f(x, y)\right\} \\
\left.=-x f_{x}^{\prime}(x, y)-y f_{y}^{\prime}(x, y)+\frac{1}{2}\left\{\left(1-x^{2}\right) f_{x x}^{\prime \prime 2}\right) f_{y y}^{\prime \prime}(x, y)\right\} .
\end{gathered}
$$

Proof. Let $(x, y) \in \mathbb{A}$ and $f \in C^{2}(\mathbb{A})$. Define the function $\psi$ by

$$
\begin{array}{cc}
\psi(t, u ; x, y) & \\
= \begin{cases}\frac{f(t, u)-f(x, y)-f_{x}(t-x)-f_{y}(u-y)-\frac{1}{2}\left\{f_{x x}(t-x)^{2}+2 f_{x y}(t-x)(u-y)+f_{y y}(u-y)^{2}\right\}}{\sqrt{(t-x)^{4}+(u-y)^{4}}}, & (t, u) \neq(x, y) \\
0 & , \quad(t, u)=(x, y) .\end{cases}
\end{array}
$$

Then by assumption we get $\psi(., . ; x, y)=\psi(.,.) \in C(\mathbb{A})$. By the Taylor formula for $f \in C(\mathbb{A})$, we have

$$
\begin{aligned}
f(t, u) & =f(x, y)+f_{x}^{\prime}(x, y)(t-x)+f_{y}^{\prime}(x, y)(u-y) \\
& +\frac{1}{2}\left\{f_{x x}^{\prime \prime}(x, y)(t-x)^{2}+2 f_{x y}^{\prime \prime}(x, y)(t-x)(u-y)+f_{y y}^{\prime \prime}(x, y)(u-y)^{2}\right\} \\
& +\psi(t, u) \sqrt{(t-x)^{4}+(u-y)^{4}}
\end{aligned}
$$


Since the operator $D_{n, n}$ is linear, we obtain

$$
\begin{aligned}
& D_{n, n}(f(t, u) ; x, y) \\
& =f(x, y)+f_{x}^{\prime}(x, y) D_{n, n}((t-x) ; x, y)+f_{y}^{\prime}(x, y) D_{n, n}((u-y) ; x, y) \\
& +\frac{1}{2}\left[f_{x x}^{\prime \prime}(x, y) D_{n, n}\left((t-x)^{2} ; x, y\right)+2 f_{x y}^{\prime \prime}(x, y) D_{n, n}((t-x) ; x, y) D_{n, n}((u-y) ; x, y)\right. \\
& \left.+f_{y y}^{\prime \prime}(x, y) D_{n, n}\left((u-y)^{2} ; x, y\right)\right] \\
& +D_{n, n}\left(\psi(t, u) \sqrt{(t-x)^{4}+(u-y)^{4}} ; x, y\right)
\end{aligned}
$$

Applying the Cauchy-Schwartz inequality for the last term on the right-hand side of (2.17), we get

$$
\begin{gathered}
\left|D_{n, n}\left(\psi(t, u) \sqrt{(t-x)^{4}+(u-y)^{4}} ; x, y\right)\right| \\
\leq\left\{D_{n, n}\left(\psi^{2}(t, u) ; x, y\right)\right\}^{\frac{1}{2}}\left\{D_{n, n}\left((t-x)^{4}+(u-y)^{4} ; x, y\right)\right\}^{\frac{1}{2}} \\
=\left\{D_{n, n}\left(\psi^{2}(t, u) ; x, y\right)\right\}^{\frac{1}{2}}\left\{D_{n, n}\left((t-x)^{4} ; x, y\right)+D_{n, n}\left((u-y)^{4} ; x, y\right)\right\}^{\frac{1}{2}}
\end{gathered}
$$

Theorem 2.2 implies

$$
\lim _{n \rightarrow \infty} D_{n, n}\left(\psi^{2}(t, u) ; x, y\right)=\psi^{2}(x, y)=0 .
$$

Using 2.18) and Lemma 2.3, we obtain

$$
\lim _{n \rightarrow \infty} n D_{n, n}\left(\psi(t, u) \sqrt{(t-x)^{4}+(u-y)^{4}} ; x, y\right)=0 .
$$

Using (2.19) and Lemma 2.1, we derive (2.16) from (2.17).

Theorem 2.5. For every $f \in C^{1}(\mathbb{A})$ such that $f_{x}, f_{y} \in C(\mathbb{A})$, we have

$$
\begin{aligned}
& \lim _{n \rightarrow \infty} \frac{\partial}{\partial x} D_{n, n}(f ; x, y)=\frac{\partial f}{\partial x}(x, y), \quad x \neq-1,1 \\
& \lim _{n \rightarrow \infty} \frac{\partial}{\partial y} D_{n, n}(f ; x, y)=\frac{\partial f}{\partial y}(x, y) \quad y \neq-1,1 .
\end{aligned}
$$

Proof. We shall prove only $(2.20)$ because the proof of $(2.21)$ is identical. Let $(x, y) \in \mathbb{A}$ for $x \neq-1,1$ be a fixed point. From $(1.3)$ it follows that

$$
\begin{aligned}
\frac{\partial}{\partial x} D_{n, n}(f(t, u) ; x, y) & =-\frac{n}{1-x} D_{n, n}(f(t, u) ; x, y) \\
& +\frac{2}{(1+x)(1-x)} D_{n, n}(k f(t, u) ; x, y), \quad \forall n \in \mathbb{N} .
\end{aligned}
$$

By the Taylor formula for $f \in C^{1}(\mathbb{A})$, we have

$$
\begin{aligned}
f(t, u) & =f(x, y)+f_{x}^{\prime}(x, y)(t-x)+f_{y}^{\prime}(x, y)(u-y) \\
& +\chi(t, u ; x, y) \sqrt{(t-x)^{2}+(u-y)^{2}}, \quad(t, u) \in \mathbb{A}
\end{aligned}
$$




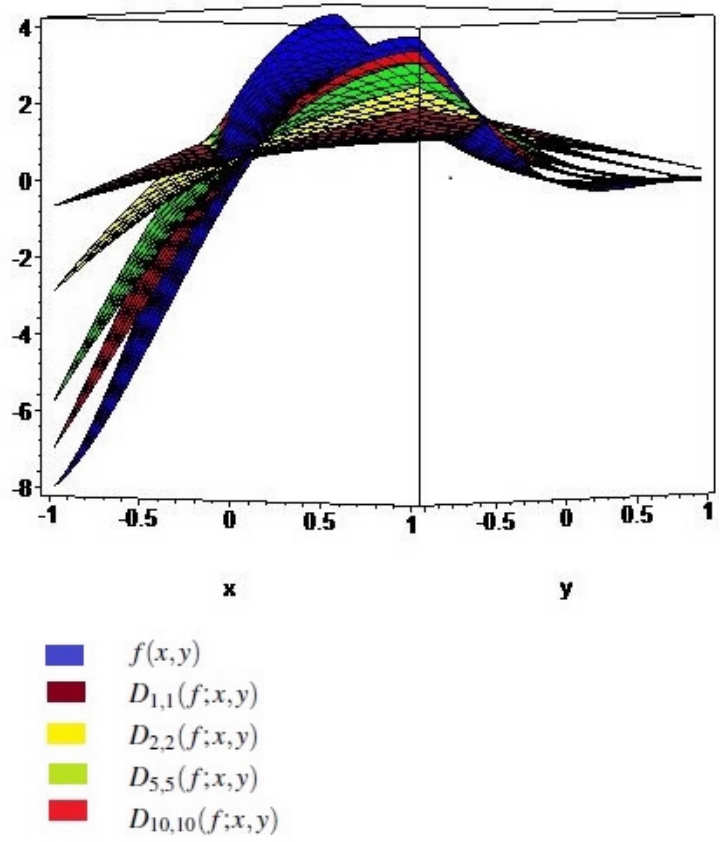

where $\chi(., . ; x, y)=\chi(.,.) \in C$ and $\chi(x, y)=0$.

From 2.1) and (2.7), we get

$$
\begin{aligned}
& \frac{\partial}{\partial x} D_{n, n}(f(t, u) ; x, y) \\
& =f(x, y)\left\{-\frac{n}{1-x} D_{n, n}(1 ; x, y)+\frac{2}{(1-x)(1+x)} D_{n, n}(k ; x, y)\right\} \\
& +f_{x}^{\prime}(x, y)\left\{-\frac{n}{1-x} D_{n, n}(t-x ; x, y)+\frac{2}{(1-x)(1+x)} D_{n, n}(k(t-x) ; x, y)\right\} \\
& +f_{y}^{\prime}(x, y)\left\{-\frac{n}{1-x} D_{n, n}(u-y ; x, y)+\frac{2}{(1-x)(1+x)} D_{n, n}(k(u-y) ; x, y)\right\} \\
& +\frac{n}{(1-x)(1+x)} D_{n, n}\left(\left(2 \frac{k}{n}-x-1\right) \chi(t, u) \sqrt{(t-x)^{2}+(u-y)^{2}} ; x, y\right)
\end{aligned}
$$

From Lemma 2.1, we obtain

$$
\begin{aligned}
\frac{\partial}{\partial x} D_{n, n}(f(t, u) ; x, y) & =\frac{n}{n+1} f_{x}^{\prime}(x, y)+\frac{n}{(1-x)(1+x)} D_{n, n}\left(\left(2 \frac{k}{n}-x-1\right)\right. \\
& \left.\times \chi(t, u) \sqrt{(t-x)^{2}+(u-y)^{2}} ; x, y\right) .
\end{aligned}
$$


Using the Cauchy-Schwartz inequality, we get

$$
\begin{aligned}
& n\left|D_{n, n}\left(\left(2 \frac{k}{n}-x-1\right) \chi(t, u) \sqrt{(t-x)^{2}+(u-y)^{2}} ; x, y\right)\right| \\
& \leq\left\{D_{n, n}\left(\left(2 \frac{k}{n}-x-1\right)^{2} ; x, y\right)\right\}^{\frac{1}{2}}\left\{n^{2} D_{n, n}\left(\chi^{2}(t, u)\left((t-x)^{2}+(u-y)^{2}\right) ; x, y\right)\right\}^{\frac{1}{2}} \\
& \leq\left\{D_{n, n}\left(\left(2 \frac{k}{n}-x-1\right)^{2} ; x, y\right)\right\}^{\frac{1}{2}}\left\{n^{2} D_{n, n}\left(\chi^{4}(t, u) ; x, y\right)\left[D_{n, n}\left((t-x)^{4}\right) ; x, y\right)\right. \\
& \left.\left.\left.\left.\left.+2 D_{n, n}\left((t-x)^{2} ; x, y\right)\right) D_{n, n}\left((u-y)^{2}\right) ; x, y\right)+D_{n, n}\left((u-y)^{4}\right) ; x, y\right)\right]\right\}^{\frac{1}{4}} .
\end{aligned}
$$

From 1.3), we obtain

$$
\begin{aligned}
D_{n, n}\left(\left(2 \frac{k}{n}-x-1\right)^{2} ; x, y\right) & =D_{n, n}\left(4 \frac{k^{2}}{n^{2}}-4 \frac{k}{n}(x+1)+(x+1)^{2} ; x, y\right) \\
& =\frac{4}{n^{2}} D_{n, n}\left(k^{2} ; x, y\right)-\frac{4}{n}(x+1) D_{n, n}(k ; x, y) \\
& +(x+1)^{2} D_{n, n}(1 ; x, y) .
\end{aligned}
$$

From (2.1) and

$$
\begin{gathered}
D_{n, n}\left(k^{2} ; x, y\right)=\frac{n(n-1)(1+x)^{2}}{4}+\frac{n(1+x)}{2}, \\
D_{n, n}(k ; x, y)=\frac{n(1+x)}{2}
\end{gathered}
$$

equalities, we get

$$
D_{n, n}\left(\left(2 \frac{k}{n}-x-1\right)^{2} ; x, y\right)=\frac{1-x^{2}}{n} .
$$

So, we obtain from Lemma 2.2 and Lemma 2.3

$$
\begin{aligned}
& n\left|D_{n, n}\left(\left(2 \frac{k}{n}-x-1\right) \chi(t, u) \sqrt{(t-x)^{2}+(u-y)^{2}} ; x, y\right)\right| \\
& \leq M(x, y) n^{-1}\left(D_{n, n}\left(\chi^{4}(t, u) ; x, y\right)\right)^{\frac{1}{4}} .
\end{aligned}
$$

From Theorem 2.2, we get

$$
\lim _{n \rightarrow \infty} D_{n, n}\left(\chi^{4}(t, u) ; x, y\right)=\chi^{4}(x, y)=0, \quad(x, y) \in \mathbb{A}
$$

which used to 2.23 gives

$$
\lim _{n \rightarrow \infty} n D_{n, n}\left(\left(2 \frac{k}{n}-x-1\right) \chi(t, u) \sqrt{(t-x)^{2}+(u-y)^{2}} ; x, y\right)=0 .
$$


Consequently, we obtain from 2.22

$$
\lim _{n \rightarrow \infty} \frac{\partial}{\partial x} D_{n, n}(f ; x, y)=\frac{\partial f}{\partial x}(x, y) .
$$

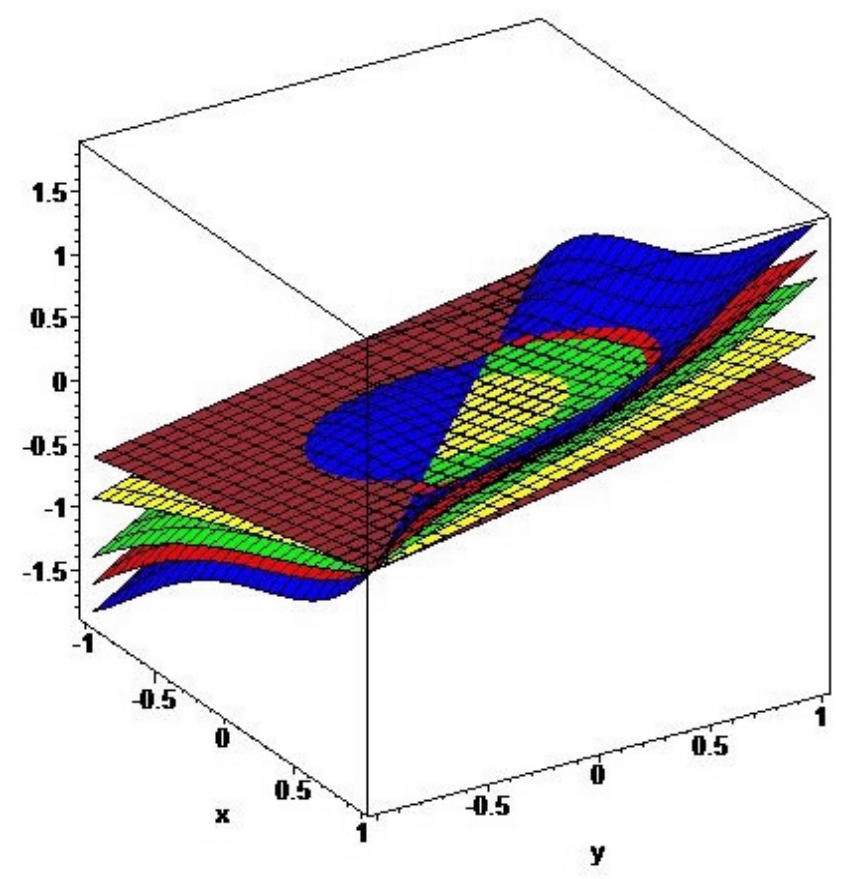

$$
\begin{aligned}
& f(x, y) \\
& D_{1,1}(f ; x, y) \\
& D_{2,2}(f ; x, y) \\
& D_{5,5}(f ; x, y) \\
& D_{10,10}(f ; x, y)
\end{aligned}
$$

Figure 1. Approximation to the function $f(x, y)=\left(x^{2}+\right.$ $\left.y^{2}\right) \sin (x+y)$ by the generalized Bernstein-Kantorovich operators.

\section{REFERENCES}

[1] Bernstein, S. N., Demonstration du theorem de Weierstrass fondee sur le calculu des probabilites, Comp. Comm. Soc. Mat. Charkow Ser., 13(2)(1912), 1-2.

[2] Korovkin, P. P., On convergence of linear positive operators in the space of continuous functions, Dokl. Akad. Nauk, 90(1953), 961-964. 
[3] Kantorovich, L. V., Sur certains developments suivant les polynomes de la forms de S. Bernstein I, II, Dokal Akad Nauk SSSR, (1930) 595-600, 563-568.

[4] Durrmeyer, J. L., Une formula d'invension de la transforms de Laplace-Appliction a'la the orie des moments, The'se de 3e cycle, Faculte' des Sciences de I'Universite de Paris, (1967).

[5] Izgi, A., Approximation by a class of new type Bernstein polynomials of one two variables, Global Journal of Pure and Applied Mathematics, 8(5) (2012), 55-71.

[6] Cao, J. D., A generalization of the Bernstein Polynomials, J. Math. Anal. Appl., 209 (1997), no.1, 140-146.

[7] Lorentz, G. G., Bernstein polynomials, Chelsea, New York, 1986.

[8] Gurdek, M., Rempulska, L. and Skorupka, M., The Baskakov operators for functions of two variables, Collect. Math., 50(3) (1999), 289-302.

[9] Kahvecibasi, I., Approximation properties of the Bernstein-Kantorovich operators on the interval [-1,1], Master of Science Thesis, Graduate School of Natural and Applied Sciences Department of Mathematic, Harran University, Sanlıurfa, Turkey.

[10] Volkov, V. I., On the convergence of sequences of linear positive operators in the space of two variables, Dokl. Akad. Nauk. SSSR (N.S.), 115 (1957), 17-19.

[11] Dirik, F. and Demirci, K., Korovkin type approximation theorem for functions of two variables in statistical sense, Turk. J. Math., 34 (2010), 73-83.

[12] Stancu, D. D., A method for obtaining polynomials of Bernstein type of two variables, Amer. Math. Monthly, 70(3) (1963), 260-264.

[13] Gazanfer, A. K. and Büyükyazici, I., Approximation by certain linear positive operators of two variables, Hindawi Publishing Corporation Abstract and Applied Analysis, ID 782080, (2014).

[14] Sahai, A., An iterative reduced-bias algorithm for a dual-fusion variant of Bernstein's operator, Inter. Journal of Math. Arch., 2(3) (2011), 331-334.

Current address: Döne Karahan: Science and Letter Faculty, Mathematics Department, Harran University Sanlıurfa TURKEY

E-mail address: dkarahan@harran.edu.tr

ORCID Address: http://orcid.org/0000-0001-6644-5596

Current address: Aydın Izgi: Science and Letter Faculty, Mathematics Department, Harran University Sanliurfa TURKEY

E-mail address: aydinizgi@yahoo.com

ORCID Address: http://orcid.org/0000-0003-3715-8621 